Published in final edited form as:

Int J Heat Mass Transf. 1978 August ; 21(8): 1133-1141. doi:10.1016/0017-9310(78)90112-6.

\title{
MASS TRANSFER IN THE ENTRANCE REGION OF A CIRCULAR TUBE
}

\author{
A. S. Popel and J. F. Gross \\ Department of Chemical Engineering, University of Arizona, Tucson, AZ 85721. U.S.A
}

\begin{abstract}
A solution in the form of an asymptotic expansion is obtained for the problem of mass transfer in the entrance region of a circular tube or flat channel for arbitrary hydrodynamically-developed velocity profile and arbitrary dependence of the diffusion coefficient on the coordinate perpendicular to the flow. Boundary conditions of the first, second and third kind are considered. The results of the analysis are compared with known approximate and numerical solutions of similar problems.
\end{abstract}

\section{INTRODUCTION}

The problem of mass transfer in the entrance region of circular tubes and flat channels with fully-developed flow has been treated by most authors as an eigenvalue problem. The solution of the Graetz-Nusselt problem for large Peclet numbers, when the axial diffusion is neglected, with the boundary condition of the first kind at the tube wall was significantly extended in [1-3] by computing large number of eigenvalues and eigenfunctions. A similar approach was employed in $[2,4,5]$ for the case of the boundary condition of the second kind. In $[3,6]$ the problem was studied for the boundary condition of the third kind in which the concentration at the tube wall is proportional to the diffusion flux normal to the wall.

Although the eigenvalue method can provide an exact solution to the mass transfer problem in the entrance region of a tube or channel, a large number of eigenvalues and eigenfunctions must be computed to obtain this solution.

Another approach to the solution of the problem was proposed by Mercer [7,8], who extended Lévêque's similarity solution [9] in the form of a power-series asymptotic expansion; only the boundary condition of the first kind was considered. Ordinary second order differential equations were derived to obtain the functional coefficients of the powerseries. However, only the first two coefficients were expressed in analytical form. A numerical solution was presented for the third and fourth terms. Worsøe-Schmidt [10] further developed Mercer's analysis and obtained numerical solutions for seven terms of this expansion. He also considered the boundary condition of the second kind and gave an analytical solution for the first term and tabulated the numerical values for the next six terms of the corresponding expansion. Newman [11] further extended the analysis of the problem for the boundary condition of the first kind by finding an analytical solution for the third term of the expansion. 
Kooijman [12] reviewed numerous solutions available in the literature and made a comparative analysis of asymptotic and numerical solutions for different types of geometries and boundary conditions. The advantage of using the series asymptotic expansions for the entrance region was shown.

In the present paper we develop a procedure which makes it possible to obtain an analytical solution for all terms of the corresponding asymptotic expansion for any of the three types of boundary conditions by solving an appropriate system of linear algebraic equations.

Therefore, solutions for the cases with boundary conditions of the first and second kind are extended and the solution for the boundary condition of the third kind is obtained for the first time. The problem of mass transfer in flow with an arbitrary fully-developed velocity profile and radial variation of the diffusion coefficient can be considered within the framework of this method.

\section{THE CONVECTIVE DIFFUSION EQUATION AND BOUNDARY CONDITIONS}

We consider the steady-state flow of fluid in a circular tube of radius $R$ with an arbitrary hydrodynamically-developed velocity profile

$$
u=u_{0} \phi\left(\frac{r}{R}\right) ; \quad \phi(0)=1, \phi(1)=0 .
$$

Assuming cylindrical symmetry of the problem and neglecting diffusion in the axial direction we can write the convective diffusion equation in the form

$$
u \frac{\partial c}{\partial z}=\frac{1}{r} \frac{\partial}{\partial r}\left(r D \frac{\partial c}{\partial r}\right)
$$

where the diffusion coefficient

$$
D=D_{0} \psi\left(\frac{r}{R}\right) ; \quad \psi(0)=1
$$

may be a function of the radial coordinate. The radial variation of the diffusion coefficient can be associated, for instance, with nonuniform fluid properties or, if the flow of a particulate medium is considered, with the diffusion augmentation due to the additional mixing by suspended particles. In the latter case, the diffusion coefficient is a function of the local shear rate $[13,14]$.

We assume a uniform concentration at the tube entrance 


$$
c=c_{0} \quad \text { at } \quad z=0 \text {, }
$$

and the symmetry condition at the axis of the tube given by

$$
\frac{\partial c}{\partial r}=0 \quad \text { at } \quad r=0 .
$$

At the tube wall, $r=R$, the boundary conditions of the first, second, and third kind are expressed as

$$
\begin{gathered}
c=c_{w} \\
-D \frac{\partial c}{\partial r}=j \\
-D \frac{\partial c}{\partial r}=k\left(c-c_{\infty}\right)
\end{gathered}
$$

where $c_{W}, j, c_{\infty}$ and $k$ are given constants.

Physically, the boundary condition (6c) may describe a first-order chemical reaction with constant reaction rate $k$, or the diffusion through the tube wall with finite conductivity of the wall. In the latter case, $c_{\infty}$ is the concentration at the outer surface of the tube wall, and the coefficient $k$ is given by

$$
k=\frac{D_{t}}{R} \ln \frac{R_{0}}{R}
$$

where $D_{t}$ is the diffusivity within the tube wall, $R_{0}$ is the outer tube radius.

We introduce the dimensionless variables and parameters 


$$
\begin{aligned}
& t=\frac{z}{R}, \quad \rho=\frac{r}{R}, \quad X=\frac{c_{0}-c}{c_{0}}, \quad X_{w}=\frac{c_{0}-c_{w}}{c_{0}}, \\
& X_{\infty}=\frac{c_{0}-c_{\infty}}{c_{0}}, \quad G=\frac{j R}{D_{w} c_{0}}, \\
& \theta=\frac{k R}{D_{w}}, \quad \varepsilon=P e^{-1}=\frac{D_{w}}{2 u_{0} R}
\end{aligned}
$$

where $D_{W}$ is the value of the diffusion coefficient at the wall $D_{W}=D_{0} \psi(1)$, and $P e$ is the Peclet number.

In terms of the variables (7) equation (2) takes the form

$$
\frac{1}{2} \phi(\rho) \frac{\partial X}{\partial t}=\frac{\varepsilon}{\rho} \frac{\partial}{\partial \rho}\left(\rho \psi(\rho) \frac{\partial X}{\partial \rho}\right) .
$$

The boundary conditions (4)-(6) in the dimensionless form are

$$
\begin{gathered}
X=0 \quad \text { at } \quad t=0 \quad \text { (9) } \\
\frac{\partial X}{\partial \rho}=0 \quad \text { at } \quad \rho=0 \\
X=X_{w} \quad(11 \mathrm{a}) \\
\frac{\partial X}{\partial \rho}=G \quad(11 \mathrm{~b}) \\
\frac{\partial X}{\partial \rho}=\theta\left(X_{\infty}-X\right) \quad \text { at } \quad \rho=1 .
\end{gathered}
$$

In particular, if a parabolic velocity profile $\phi=1-\rho^{2}$ and constant diffusion coefficient $\psi=$ 1 are considered, equation (8) becomes 


$$
\frac{1}{2}\left(1-\rho^{2}\right) \frac{\partial X}{\partial t}=\frac{\varepsilon}{\rho} \frac{\partial}{\partial \rho}\left(\rho \frac{\partial X}{\partial \rho}\right) .
$$

For large Peclet numbers, the parameter $\mathcal{\varepsilon}$ is small and therefore we have a singular perturbation problem.

A certain generalization of equation (8) is now considered and an asymptotic solution to this equation is derived.

\section{ASYMPTOTIC SOLUTION OF A CERTAIN PARABOLIC DIFFERENTIAL EQUATION WITH SMALL PARAMETER $\varepsilon$}

Equation (8) is a particular case of a more general linear parabolic differential equation

$$
y^{m-2} h(y) \frac{\partial X}{\partial t}=\varepsilon\left[\frac{\partial^{2} X}{\partial y^{2}}+f(y) \frac{\partial X}{\partial y}+g(y) X\right]
$$

where $m \geq 2$ is integer, $t \geq 0$ and $0 \leq y \leq 1$. The coefficients $h, f$ and $g$ in equation (13) are analytic functions of $y$ at $0 \leq y<1$; (8) can be written in the form (13) by transformation of the radial coordinate $y=1-\rho$.

Assuming $h(0) \neq 0$ without loss of generality one can set $h(0)=1$.

At $t=0$ the boundary condition (9) is considered, whereas (10) can be replaced by less restrictive condition:

$$
|X|<\infty \quad \text { at } \quad 0 \leq y \leq 1 .
$$

At $y=0$ one of the following conditions is to be fulfilled

$$
\begin{gathered}
X=X_{w} \\
\frac{\partial X}{\partial y}=-G \\
\frac{\partial X}{\partial y}=\theta\left(X-X_{\infty}\right) .
\end{gathered}
$$


For small values of the parameter $\varepsilon$ a solution of the problem will be sought in the form of an asymptotic expansion by using the singular perturbation technique.

It is apparent that the outer solution of equation (13) with boundary conditions (9) and (14) is

$$
X(t, y)=0 .
$$

In order to satisfy one of the boundary conditions (15) the inner coordinate

$$
\xi=\varepsilon^{-v} y
$$

is introduced with $v=1 / m$.

In terms of the new inner variable, equations (13) and (15) become

$$
\xi^{m-2} h \frac{\partial X}{\partial t}=\frac{\partial^{2} X}{\partial \xi^{2}}+\varepsilon^{v} f \frac{\partial X}{\partial \xi}+\varepsilon^{2 v} g X
$$

$$
\begin{gathered}
X=X_{w} \quad(19 \mathrm{a}) \\
\frac{\partial X}{\partial \xi}=-\varepsilon^{v} G \quad(19 \mathrm{~b}) \\
\frac{\partial X}{\partial \xi}=\varepsilon^{v} \theta\left(X-X_{\infty}\right) \text { at } \xi=0
\end{gathered}
$$

where $h, f$ and $g$ are now functions of the variable $\varepsilon^{v} \xi$.

The inner solution is expressed in the form

$$
X=\sum_{n=0}^{\infty} \varepsilon^{n v} X_{n} .
$$

The application of the Laplace transform 


$$
\bar{X}(s, \xi)=\int_{0}^{\infty} X(t, \xi) e^{-s t} \mathrm{~d} t
$$

to (18) and (19) after replacing $\xi$ by

$$
\eta=s^{v} \xi
$$

yields

$$
\begin{gathered}
\eta^{m-2} h \bar{X}=\bar{X},_{\eta \eta}+\left(\frac{\varepsilon}{s}\right)^{v} f \bar{X}_{{ }_{\eta}}+\left(\frac{\varepsilon}{s}\right)^{2 v} g \bar{X} \\
\bar{X}=\frac{X_{w}}{s} \quad(22 \mathrm{a}) \\
\bar{X},_{\eta}=-\left(\frac{\varepsilon}{s}\right)^{v} \frac{G}{s} \quad(22 \mathrm{~b}) \\
X,_{\eta}=\left(\frac{\varepsilon}{s}\right)^{v} \theta\left(\bar{X}-\frac{X_{\infty}}{s}\right) \text { at } \eta=0
\end{gathered}
$$

where the subscript ", $\eta$ " denotes the differentiation with respect to $\eta$. The functions $h, f$ and $g$ can be represented by Taylor series as

$$
\begin{aligned}
& h=\sum_{k=0}^{\infty}\left(\frac{\varepsilon}{s}\right)^{k v} h_{k} \eta^{k}, \quad f=\sum_{k=0}^{\infty}\left(\frac{\varepsilon}{s}\right)^{k v} f_{k} \eta^{k}, \\
& g \sum_{k=0}^{\infty}\left(\frac{\varepsilon}{s}\right)^{k v} g_{k} \eta^{k}
\end{aligned}
$$

where $h_{k}, f_{k}$ and $g_{k}$ are constants.

After substitution of (23) and (19) into (21) and equating powers of $\varepsilon^{v}$ we obtain a set of inhomogeneous ordinary differential equations

Int J Heat Mass Transf. Author manuscript; available in PMC 2018 January 17. 


$$
\bar{X}_{n, \eta \eta}-\eta^{m-2} \bar{X}_{n}=\sum_{k=1}^{n} h_{k} s^{-k v} \eta^{k+m-2} \bar{X}_{n-k}-\sum_{k=0}^{n-1} f_{k} s^{-(k+1) v} \eta^{k} \bar{X}_{n-k-1, \eta}-\sum_{k=0}^{n-2} g_{k} s^{-(k+2) v} \eta^{k} \bar{X}_{n-k-2}
$$

where $h_{0}=1$.

The boundary conditions (22) yield

$$
\begin{gathered}
\bar{X}_{0}=X_{w} / s, \bar{X}_{n}=0 \text { for } n \geq 1 \text { at } \eta=0 \\
\bar{X}_{1, \eta}=-G / s^{1+v}, \bar{X}_{n, \eta}=0 \text { for } n=0, n \geq 2 \text { at } \eta=0 \\
\bar{X}_{0, \eta}=0, \bar{X}_{1, \eta}=\frac{\theta}{s^{v}}\left(\bar{X}_{0}-\frac{X_{\infty}}{s}\right), \bar{X}_{n, \eta}=\frac{\theta}{s^{v}} \bar{X}_{n-1} \text { for } n \geq 2 \text { at } \eta=0 .
\end{gathered}
$$

The matching condition with the outer solution (16), makes it possible to infer that

$$
\bar{X}_{n} \rightarrow 0 \quad \text { as } \quad \eta \rightarrow \infty
$$

The general bounded solution to the homogeneous equation for $\bar{X}_{n}$

$$
\bar{X}_{n, \eta \eta}-\eta^{m-2} \bar{X}_{n}=0
$$

corresponding to the inhomogeneous equation (24), is [15]

$$
\bar{X}_{n}=A_{n}^{0} \eta^{1 / 2} K_{v}\left(\frac{2}{m} \eta^{m / 2}\right)
$$

where $K_{v}$ is the modified Bessel function of the second kind.

We now seek a bounded solution of (24) in the form 


$$
\bar{X}_{n}=s^{-(1+n v)}\left[\eta^{1 / 2} K_{v}\left(\frac{2}{m} \eta^{m / 2}\right) \sum_{r=0}^{N} A_{n}^{r} \eta^{r}+\eta^{(m-1) / 2} K_{1-v}\left(\frac{2}{m} \eta^{m / 2}\right) \sum_{r=0}^{M} B_{n}^{r} \eta^{r}\right]
$$

where the upper indices $N$ and $M$ in the summations over $r$ are finite numbers and will be determined in the process of solution.

With the help of the following relationships which can easily be proved by direct differentiation and using recurrence relations for Bessel functions [15]

$$
\begin{aligned}
& \frac{\mathrm{d}}{\mathrm{d} \eta}\left[\eta^{1 / 2} K_{v}\left(\frac{2}{m} \eta^{m / 2}\right)\right]=-\eta^{(m-1) / 2} K_{1-v}\left(\frac{2}{m} \eta^{m / 2}\right) \\
& \frac{\mathrm{d}}{\mathrm{d} \eta}\left[\eta^{(m-1) / 2} K_{1-v}\left(\frac{2}{m} \eta^{m / 2}\right)\right]=-\eta^{m-3 / 2} K_{v}\left(\frac{2}{m} \eta^{m / 2}\right)
\end{aligned}
$$

the first and second derivatives of (29) are calculated. Substitution into (24) yields a system of linear algebraic equations for $A_{n}^{r}$ and $B_{n}^{r}$ :

$$
\begin{aligned}
& r(r-1) A_{n}^{r}-(2 r-m) B_{n}^{r-m+1}=\sum_{k=1}^{n} h_{k} A_{n-k}^{r-k-m}-\sum_{k=0}^{n-1} f_{k}\left[(r-k-1) A_{n-k-1}^{r-k-1}-B_{n-k-1}^{r-k-m}\right]-\sum_{k=0}^{n-2} g_{k} A_{n-k-2}^{r-k-2}, r \geq 2 \\
& -2 r A_{n}^{r}+r(r+1) B_{n}^{r+1}=\sum_{k=1}^{n} h_{k} B_{n-k}^{r-k-m+1}+\sum_{k=0}^{n-1} f_{k}\left[A_{n-k-1}^{r-k-1}-(r-k) \sum_{k=0}^{n-2} g_{k} B_{n-k-2}^{r-k-1}, r \geq 1 .\right.
\end{aligned}
$$

Equations (31) can be considered as recursion relationships since they express the coefficients $A_{n}^{r}$ and $B_{n}^{r}$ in terms of $A_{p}^{l}$ and $B_{p}^{l}$ with $p=0,1, \ldots, n-1$.

It should be noted that for a given $n$, by solving the system of equations (31) one determines all coefficients with subscript $n$, except $A_{n}^{0}$. These coefficients will be determined below by using one of the boundary conditions (25).

The expansions of the Bessel functions $K_{v}$ and $K_{1-\nu}$, at $\eta=0$ read [15]

$$
\begin{aligned}
& \eta^{1 / 2} K_{v}\left(\frac{2}{m} \eta^{m / 2}\right)=\frac{\pi}{2 \sin (\pi v)}\left[\frac{v^{-v}}{\Gamma(1-v)}-\frac{v^{v} \eta}{\Gamma(1+v)}+O\left(\eta^{m}\right)\right] \\
& \eta^{(m-1) / 2} K_{1-v}\left(\frac{2}{m} \eta^{m / 2}\right)=\frac{\pi}{2 \sin (\pi v)}\left[\frac{v^{-(1-v)}}{\Gamma(v)}+O\left(\eta^{m-1}\right)\right] .
\end{aligned}
$$


Hence the solution (29) can be rewritten for small $\eta$ and $m \geq 3$ as

$$
\bar{X}_{n}=\mu_{v} S^{-(1+n v)}\left[A_{n}^{0}+\lambda_{v} B_{n}^{0}+\eta\left(A_{n}^{1}-\lambda_{v} A_{n}^{0}+\lambda_{v} B_{n}^{1}\right)+O\left(\eta^{2}\right)\right]
$$

where

$$
\lambda_{v}=v^{2 v} \frac{\Gamma(1-v)}{\Gamma(1+v)}, \mu_{v}=\frac{\pi}{2 \sin (\pi v) v^{v} \Gamma(1-v)} .
$$

When $m=2$, the form of the solution can be greatly simplified since

$$
\eta^{1 / 2} K_{1 / 2}(\eta)=\left(\frac{\pi}{2}\right)^{1 / 2} \mathrm{e}^{-\eta}
$$

Since the function $\overline{X_{0}}$ satisfies the homogeneous equation (27) and therefore is given by (28), we infer $B_{0}^{0}=0$. For the boundary condition of the first kind, it follows from (25a) and (33) that

$$
A_{0}^{0}=X_{w} / \mu_{v}, \quad A_{n}^{0}=-\lambda_{v} B_{n}^{0} \text { for } n \geq 1
$$

Similarly, it can be shown, for the boundary condition of the second kind (25b) that

$$
A_{0}^{0}=0, \quad A_{1}^{0}=G / \mu_{v}, A_{n}^{0}=\left(A_{n}^{1}+\lambda_{v} B_{n}^{1}\right) / \lambda \quad \text { for } \quad n \geq 2
$$

and for the boundary condition of the third kind $(25 \mathrm{c})$ :

$$
A_{0}^{0}=0, \quad A_{1}^{0}=X_{\infty} \theta / \mu_{v}, \quad A_{n}^{0}=\left[A_{n}^{1}+\lambda_{v} B_{n}^{1}-\theta\left(A_{n-1}^{0}+\lambda_{v} B_{n-1}^{0}\right)\right] / \lambda_{v} \quad \text { for } \quad n \geq 2 .
$$

Therefore, as soon as the system of algebraic equations (31) for a certain $n$ is solved, the Laplace transform $\bar{X}_{n}$ for the corresponding term in the asymptotic expansion (19) is known. Equation (33) yields 


$$
\begin{aligned}
& \bar{X}_{n}(s, 0)=\frac{\mu_{v}}{s^{1+n v}}\left(A_{n}^{0}+\lambda_{v} B_{n}^{0}\right) \\
& \frac{\partial \bar{X}_{n}(s, 0)}{\partial y}=\frac{\mu_{v}}{\varepsilon^{v} s^{1+(n-1) v}}\left(A_{n}^{1}-\lambda_{v} A_{n}^{0}+\lambda_{v} B_{n}^{1}\right) .
\end{aligned}
$$

Inverse Laplace transforms of (39) give the values of the function $X(t, 0)$ and the gradient normal to the boundary $\partial X(t, 0) / \partial y$

$$
\begin{gathered}
X(t, 0)=\mu_{v} \sum_{n=0}^{\infty} \frac{(\varepsilon t)^{n v}}{\Gamma(1+n v)}\left(A_{n}^{0}+\lambda_{v} B_{n}^{0}\right) \\
\frac{\partial X(t, 0)}{\partial y}=\mu_{v} \sum_{n=0}^{\infty} \frac{(\varepsilon t)^{(n-1) v}}{\Gamma[1+(n-1) v]} \times\left(A_{n}^{1}-\lambda_{v} A_{n}^{0}+\lambda_{v} B_{n}^{1}\right) .
\end{gathered}
$$

In order to invert the Laplace transform (29) we return to the variable $\xi$ :

$$
\bar{X}_{n}=s^{-(1+n v)}\left[K_{v}(2) v \xi^{m / 2} s^{1 / 2} \times \sum_{r} A_{n}^{r} \xi^{r+1 / 2} s^{(r+1 / 2) v}+K_{1-v}\left(2 v \xi^{m / 2} s^{1 / 2}\right) X \sum_{r} B_{n}^{r} \xi^{r+[(m-1) / 2]} s^{(r-1 / 2) v+1 / 2}\right] .
$$

Using the relationship [16]

$$
2 \alpha^{1 / 2} s^{\mu-1} K_{v}\left(2 \alpha^{1 / 2} s^{1 / 2}\right) \div t^{1 / 2-\mu} e^{-\alpha / 2 t} W_{\mu-1 / 2, v / 2}\left(\frac{\alpha}{t}\right)
$$

where $W$ is the Whittaker function, and expressing the Whittaker function in terms of the confluent hypergeometric function $\Psi$ [15]

$$
W_{\mu, v}(\zeta)=e^{-\zeta / 2} \zeta^{1 / 2+v} \Psi\left(v-\mu+\frac{1}{2}, 2 v+1 ; \zeta\right)
$$

after some appropriate calculations, we obtain the inverse Laplace transform of the solution (29) in the form 


$$
\begin{aligned}
& X(t, \xi)=\frac{1}{2} e^{-\zeta} \sum_{n=0}^{\infty}(\varepsilon t)^{n v} \sum_{r} v^{-(2 r+1) v} \zeta^{r v} \\
& \left\{A_{n}^{r} \Psi[(n-r-1) v+1,1-v ; \zeta]+v^{2 v-1} B_{n}^{r} \Psi[(n-r+1) v, v ; \zeta]\right\} .
\end{aligned}
$$

Here $\zeta$ is the similarity variable

$$
\zeta=\frac{v^{2} \xi^{m}}{t}=\frac{v^{2} y^{m}}{\varepsilon t} .
$$

It is worth mentioning that when $(n-r \pm 1) v$ in (45) is an integer, the corresponding hypergeometric function is expressed in terms of Laguerre polynomials [15].

\section{MASS TRANSFER IN THE ENTRANCE REGION OF A CIRCULAR TUBE WITH PARABOLIC VELOCITY PROFILE AND LINEAR RADIAL VARIATION OF THE DIFFUSION COEFFICIENT}

We now consider solutions of equation (8) with $\phi=1-\rho^{2}, \psi=1+\beta \rho$ with the boundary conditions (9)-(14); when $\beta=0$, (8) reduces to (12).

In order to use the general solution obtained above, equation (8) is rewritten in the form (13) by the transformation $y=1-\rho$; this yields

$$
\begin{aligned}
& m=3 ; h_{0}=1, \quad h_{1}=\bar{\beta}-\frac{1}{2}, \quad h_{k}=\bar{\beta}^{k-1}\left(\bar{\beta}-\frac{1}{2}\right) \text { as } k \geq 2 ; \\
& f_{k}=-\left(1+\bar{\beta}^{k+1}\right) \text { as } k \geq 0 ; g_{k}=0 \text { as } k \geq 0
\end{aligned}
$$

where

$$
\bar{\beta}=\frac{\beta}{1+\beta} .
$$

The expansion (45) in the case $m=3$ takes the form

$$
\begin{aligned}
& X(t, \xi)=\frac{1}{2} \mathrm{e}^{-\zeta} \sum_{n=0}^{\infty}(\varepsilon t)^{n / 3} \sum_{r} 3^{(2 r+1) / 3} \zeta^{r / 3} \\
& {\left[A_{n}^{r} \Psi\left(\frac{n-r-1}{3}, \frac{2}{3} ; \zeta\right)+3^{1 / 3} B_{n}^{r} \Psi\left(\frac{n-r+1}{3}, \frac{1}{3} ; \zeta\right)\right]}
\end{aligned}
$$


where

$$
\zeta=\frac{\xi^{3}}{9 t}=\frac{(1-\rho)^{3}}{9 \varepsilon t} .
$$

The dimensionless parameter $\tau=\varepsilon t$ expressed in terms of dimensional variables is

$$
\tau=\frac{D_{w} z}{2 u_{0} R} .
$$

(i) Boundary condition of the first kind. Uniform diffusion coefficient $(\beta=0)$. The expression for the first coefficient is given by (36):

$$
A_{0}^{0}=3^{1 / 6} \pi^{-1} \Gamma\left(\frac{2}{3}\right) X_{w} .
$$

Since $B_{0}^{0}=0$, the zero-order term in (49) assumes the form

$$
X_{0}=\frac{1}{2} \mathrm{e}^{-\zeta} 3^{1 / 3} A_{0}^{0} \Psi\left(\frac{2}{3}, \frac{2}{3} ; \zeta\right)=\frac{X_{w}}{\Gamma\left(\frac{4}{3}\right)} \int_{\zeta^{1 / 3}}^{\infty} \exp \left(z^{3}\right) \mathrm{d} z
$$

which is Lévêque's self-similar solution [9].

We introduce the notation

$$
\alpha_{n}^{r}=\frac{A_{n}^{r}}{A_{0}^{0}}, \quad \beta_{n}^{r}=\frac{B_{n}^{r}}{A_{0}^{0}}
$$

and, solving the algebraic system (31) together with (36) for $n=0,1,2,3$, find

$$
\begin{aligned}
& \alpha_{0}^{0}=1 ; \alpha_{1}^{1}=\frac{3}{5}, \beta_{1}^{2}=\frac{1}{10} ; \alpha_{2}^{0}=-\frac{11}{35} \lambda, \alpha_{2}^{2}=\frac{16}{35}, \\
& \alpha_{2}^{5}=\frac{1}{200}, \beta_{2}^{0}=\frac{11}{35}, \beta_{2}^{3}=\frac{1}{14} ; \alpha_{3}^{1}=-\frac{33}{175} \lambda, \\
& \alpha_{3}^{3}=\frac{1291}{3150}, \quad \alpha_{3}^{6}=\frac{167}{42000}, \quad \beta_{3}^{1}=\frac{661}{1575} \\
& \beta_{3}^{2}=-\frac{11}{350} \lambda, \quad \beta_{3}^{4}=\frac{173}{3150}, \beta_{3}^{7}=\frac{1}{6000}
\end{aligned}
$$

where 


$$
\lambda=\lambda_{1 / 3}=\frac{\Gamma\left(\frac{2}{3}\right)}{9^{1 / 3} \Gamma\left(\frac{4}{3}\right)}=0.729011 .
$$

For the values $n$ considered all other coefficients (53) equal zero.

The local Sherwood number, which referred to the concentration difference in the inlet, is defined as

$$
S h_{1}=\frac{j R}{D_{0}\left(c_{0}-c_{w}\right)}
$$

where $j=-D_{0} \partial c / \partial r$ is the diffusion flux at the tube wall. Using the general expression (41) one obtains

$$
S h_{1}=-\sum_{n=0}^{\infty} \frac{\tau^{(n-1) / 3}}{\Gamma\left(\frac{n+2}{3}\right)}\left(\alpha_{n}^{1}-\lambda \alpha_{n}^{0}+\lambda \beta_{n}^{1}\right)
$$

or, after substitution of the calculated values (54)

$$
S h_{1}=0.538366 \tau^{-1 / 3}-0.6-0.187047 \tau^{1 / 3}-0.186634 \tau^{2 / 3}+O(\tau)
$$

which coincides with the numerical solution obtained by Worsøe-Schmidt [10].

The average Sherwood number is introduced by

$$
\langle S h\rangle_{1}=\frac{I}{\pi z D_{0}\left(c_{0}-c_{w}\right)}
$$

where the total diffusion flux over the length $z$ is

$$
I=2 \pi R \int_{0}^{z} j \mathrm{~d} z
$$

From (55), (58) and (59) we get 


$$
\langle S h\rangle_{1}=\frac{2}{\tau} \int_{0}^{\tau} S h \mathrm{~d} \tau
$$

and, employing (57)

$$
\langle S h\rangle_{1}=1.615098 \tau^{-1 / 3}-1.2-0.280571 \tau^{1 / 3}-0.223960 \tau^{2 / 3}+O(\tau) .
$$

The first three terms in (61) coincide with the corresponding three-terms solution obtained by Newman [11].

It was shown in [11] by comparison with the eigenvalue solution that the three-term asymptotic solution was accurate to $0.1 \%$ for $\tau=0.005$. Calculations show that the accuracy of the present four-term solution is $0.01 \%$ for the same value of $\tau$.

The mixing-cup concentration $c$ for an arbitrary cross-section is determined by the relation

$$
I=Q\left(c_{0}-\langle c\rangle\right) \quad(62)
$$

where $Q$ is the constant volumetric flow rate; for the parabolic velocity profile $Q=\pi u_{0} R^{2} / 2$. Expressions (59) and (62) imply that the expression for the mixing-cup concentration is

$$
\frac{\langle c\rangle_{1}}{c_{0}}=1-4 X_{w} \tau\langle S h\rangle_{1}
$$

where $\langle S h\rangle_{1}$ given by (61).

Finally, we introduce a parameter

$$
\frac{\delta_{1}}{R}=\frac{D_{0}\left(c_{0}-c_{w}\right)}{j R}
$$

which can be considered as the dimensionless thickness of the diffusion boundary layer [17]. It follows from (55) that

$$
\frac{\delta_{1}}{R}=S h_{1}^{-1} .
$$


The leading terms in the expansions (63) and (65) can be written out by using (61) and (57), respectively

$$
\begin{gathered}
\frac{\langle c\rangle_{1}}{c_{0}}=1-6.460392 X_{w} \tau^{2 / 3}+O(\tau) \\
\frac{\delta_{1}}{R}=1.857473 \tau^{1 / 3}+O\left(\tau^{2 / 3}\right) .
\end{gathered}
$$

(ii) Boundary condition of the first kind. Variable diffusion coefficient $(\beta \neq 0)$. Equation (52) is valid in this case; the solution of (31) for $n=0,1,2$ with the notations given in (53) yields

$$
\begin{aligned}
& \alpha_{0}^{0}=1 ; \alpha_{1}^{1}=\frac{3}{5}\left(1+\frac{\bar{\beta}}{2}\right), \beta_{1}^{2}=\frac{1}{5}\left(\frac{1}{2}-\bar{\beta}\right) ; \\
& \alpha_{2}^{0}=-\frac{\lambda}{35}\left(11-19 \bar{\beta}+\frac{11}{4} \bar{\beta}^{2}\right), \\
& \alpha_{2}^{2}=\frac{1}{7}\left(\frac{16}{5}+\frac{5}{4} \bar{\beta}+\frac{53}{40} \bar{\beta}^{2}\right), \alpha_{2}^{5}=\frac{1}{50}\left(\frac{1}{2}-\bar{\beta}\right)^{2}, \\
& \beta_{2}^{0}=\frac{1}{35}\left(11-19 \bar{\beta}+\frac{11}{4} \bar{\beta}^{2}\right), \\
& \beta_{2}^{3}=\frac{1}{14}\left(1-\frac{9}{10} \bar{\beta}-\frac{11}{5} \bar{\beta}^{2}\right) .
\end{aligned}
$$

If $\beta=0$ the corresponding coefficients (68) coincide with those given by (54).

It should be noted that the relations (55), (56), (58), (60), (63), (64) and (65) of the previous section (i) are valid if $D_{0}$ is replaced by $D_{W}=(1+\beta) D_{0}$ and if the definition of $\mathcal{\varepsilon}(7)$ is considered.

Figure 1 illustrates the variation of the functions $c / c_{0}$ and $\delta / R$ vs $\beta$ for several values of the dimensionless axial coordinate $\tau$.

(iii) Boundary condition of the second kind. Uniform diffusion coefficient $(\beta=0)$.

The general expression (37) for $m=3$ reduces to

$$
A_{1}^{0}=3^{1 / 6} \pi^{-1} \Gamma\left(\frac{1}{3}\right) G \text {. }
$$

With the notation 


$$
\alpha_{n}^{r}=\frac{A_{n}^{r}}{A_{1}^{0}}, \quad \beta_{n}^{r}=\frac{B_{n}^{r}}{A_{1}^{0}}
$$

the solution of (31) together with the relationships (37) for $n=1,2,3,4$ has the form

$$
\begin{aligned}
& \alpha_{1}^{0}=1 ; \alpha_{2}^{0}=\frac{3}{5 \lambda}, \alpha_{2}^{1}=\frac{3}{5}, \beta_{2}^{2}=\frac{1}{10}, \alpha_{3}^{0}=\frac{9}{25 \lambda^{2}}, \\
& \alpha_{3}^{1}=\frac{9}{25 \lambda}, \alpha_{3}^{2}=\frac{16}{35}, \alpha_{3}^{5}=\frac{1}{200}, \beta_{3}^{0}=\frac{11}{35}, \\
& \beta_{3}^{2}=\frac{3}{50 \lambda}, \beta_{3}^{3}=\frac{1}{14}, \alpha_{4}^{0}=\frac{661}{1575}+\frac{27}{125 \lambda^{3}}, \\
& \alpha_{4}^{1}=\frac{27}{125 \lambda^{2}}, \alpha_{4}^{2}=\frac{48}{175 \lambda}, \alpha_{4}^{3}=\frac{1291}{3150}, \alpha_{4}^{5}=\frac{3}{1000 \lambda}, \\
& \alpha_{4}^{6}=\frac{167}{42000}, \beta_{4}^{0}=\frac{33}{175 \lambda}, \beta_{4}^{1}=\frac{661}{1575}, \\
& \beta_{4}^{2}=\frac{9}{250 \lambda^{2}}, \beta_{4}^{3}=\frac{3}{70 \lambda}, \beta_{4}^{4}=\frac{173}{3150}, \beta_{4}^{7}=\frac{1}{6000} .
\end{aligned}
$$

The concentration at the tube wall can now be calculated by using the general solution (40)

$$
\frac{c_{w 2}}{c_{0}}=1-\frac{G}{\lambda} \sum_{n=1}^{\infty} \frac{\tau^{n / 3}}{\Gamma\left(1+\frac{n}{3}\right)}\left(\alpha_{n}^{0}+\lambda \beta_{n}^{0}\right) .
$$

Substitution of (71) into (72) then yields

$$
\frac{c_{w 2}}{c_{0}}=1-G\left[1.536117 \tau^{1 / 3}+1.250598 \tau^{2 / 3}+1.243466 \tau+1.343060 \tau^{4 / 3}+O\left(\tau^{5 / 3}\right)\right] .
$$

The coefficients in (73) coincide with those obtained numerically by Worsøe-Schmidt [10]. If the average Sherwood number is defined as

$$
\langle S h\rangle_{2}=\frac{I}{\pi z j}
$$

then calculations yield $\langle S h\rangle_{2}=2$ and therefore the expression for the mixing-cup concentration (62) assumes the form, analogous to (63), namely 


$$
\frac{\langle c\rangle_{2}}{c_{0}}=1-4 G \tau\langle s h\rangle_{2} .
$$

In this case, the mixing-cup concentration is a linear function of the axial coordinate.

The dimensionless diffusion boundary-layer thickness, defined independently of boundary conditions by (64), with the help of (73) to the lowest order in $\tau$ is

$$
\frac{\delta_{2}}{R}=1.536117 \tau^{1 / 3}+O\left(\tau^{2 / 3}\right) .
$$

(iv) Boundary condition of the third kind. Uniform diffusion coefficient $(\beta=0)$. The relationship (38) gives

$$
A_{1}^{0}=3^{1 / 6} \pi^{-1} \Gamma\left(\frac{2}{3}\right) X_{\infty} \theta \lambda^{-1} .
$$

With

$$
\alpha_{n}^{r}=\frac{A_{n}^{r}}{A_{1}^{0}}, \quad \beta_{n}^{r}=\frac{B_{n}^{r}}{A_{1}^{0}}
$$

the solution of (31) and (38) for $n=1,2,3,4$ yields 


$$
\begin{aligned}
& \alpha_{1}^{0}=1 ; \alpha_{2}^{0}=-\frac{1}{\lambda}\left(\theta-\frac{3}{5}\right), \alpha_{2}^{1}=\frac{3}{5}, \beta_{2}^{2}=\frac{1}{10} ; \\
& \alpha_{3}^{0}=\frac{1}{\lambda^{2}}\left(\theta-\frac{3}{5}\right)^{2}, \alpha_{3}^{1}=-\frac{3}{5 \lambda}\left(\theta-\frac{3}{5}\right), \alpha_{3}^{2}=\frac{16}{35}, \\
& \alpha_{3}^{5}=\frac{1}{200}, \beta_{3}^{0}=\frac{11}{35}, \beta_{3}^{2}=-\frac{1}{10 \lambda}\left(\theta-\frac{3}{5}\right), \beta_{3}^{3}=\frac{1}{14}, \\
& \alpha_{4}^{0}=-\frac{1}{\lambda^{3}}\left[\left(\theta-\frac{3}{5}\right)^{3}+\frac{11}{35} \lambda^{3} \theta-\frac{661}{1575} \lambda^{3}\right], \\
& \alpha_{4}^{1}=\frac{3}{5 \lambda^{2}}\left(\theta-\frac{3}{5}\right)^{2}, \alpha_{4}^{2}=-\frac{16}{35 \lambda}\left(\theta-\frac{3}{5}\right), \alpha_{4}^{3}=\frac{1291}{3150}, \\
& \alpha_{4}^{5}=-\frac{1}{200 \lambda}\left(\theta-\frac{3}{5}\right), \alpha_{4}^{6}=\frac{167}{42000}, \\
& \beta_{4}^{0}=-\frac{11}{35 \lambda}\left(0-\frac{3}{5}\right), \\
& \beta_{4}^{1}=\frac{661}{1575}, \beta_{4}^{2}=\frac{1}{10 \lambda^{2}}\left(\theta-\frac{3}{5}\right)^{2}, \beta_{4}^{3}=-\frac{1}{14 \lambda}\left(\theta-\frac{3}{5}\right), \\
& \beta_{4}^{4}=\frac{173}{3150}, \beta_{4}^{7}=\frac{1}{6000} .
\end{aligned}
$$

The concentration distribution at the tube wall follows from (40)

$$
\frac{c_{w 3}}{c_{0}}=1-\frac{X_{\infty} \theta}{\lambda} \sum_{n=1}^{\infty} \frac{\tau^{n / 3}}{\Gamma\left(1+\frac{n}{3}\right)}\left(\alpha_{n}^{0}+\lambda \beta_{n}^{0}\right) .
$$

By taking (79) into account, equation (80) can be rewritten as

$$
\frac{c_{w 3}}{c_{0}}=1+\frac{X_{\infty} \theta}{\lambda} \sum_{n=0}^{\infty}(-1)^{n} T_{n} \frac{\tau^{n / 3}}{\Gamma\left(1+\frac{n}{3}\right)}
$$

where

$$
\begin{aligned}
& T_{1}=1, T_{2}=\frac{1}{\lambda}(\theta-0.6), \\
& T_{3}=\frac{1}{\lambda^{2}}\left(\theta^{2}-1.2 \theta+0.481767\right), \\
& T_{4}=\frac{1}{\lambda_{3}}\left(\theta^{3}-1.8 \theta^{2}+1.323533 \theta-0.500368\right)
\end{aligned}
$$

are polynomials of $\theta$. 
The local Sherwood number, which referred to the concentration difference at the inlet, assumes the form

$$
S h_{3}=\frac{j \cdot R}{D_{0}\left(c_{0}-c_{\infty}\right)}=\theta\left[1+\frac{\theta}{\lambda} \sum_{n=1}^{\infty}(-1)^{n} T_{n} \frac{\tau^{n / 3}}{\Gamma\left(1+\frac{n}{3}\right)}\right]
$$

so that $S h_{3}=\theta$ at $\tau=0$.

Defining the average Sherwood number by

$$
\langle S h\rangle_{3}=\frac{I}{\pi z D_{0}\left(c_{0}-c_{\infty}\right)}
$$

and using (83) we infer that

$$
\begin{aligned}
& \langle S h\rangle_{3}=\frac{2}{\tau} \int_{0}^{\tau} S h \mathrm{~d} \tau \\
& =2 \theta\left[1+\frac{\theta}{\lambda} \sum_{n=1}^{\infty}(-1)^{n} T_{n} \frac{\tau^{n / 3}}{\Gamma\left(2+\frac{n}{3}\right)}\right] .
\end{aligned}
$$

The mixing-cup concentration in (62) with the help of (84) can be expressed as

$$
\frac{\langle c\rangle_{3}}{c_{0}}=1-4 X_{\infty} \tau\langle S h\rangle_{3}
$$

which is similar to the relations (63) and (75).

The dimensionless diffusion boundary-layer thickness (64) equals

$$
\frac{\delta_{3}}{R}=\frac{1}{S h_{3}}-\frac{1}{\theta}
$$

The leading terms in the expansions (80), (86) and (87) can be easily calculated

$$
\frac{c_{w 3}}{c_{0}}=1-1.536117 X_{\infty} \theta \tau^{1 / 3}+O\left(\tau^{2 / 3}\right)
$$

Int J Heat Mass Transf. Author manuscript; available in PMC 2018 January 17. 


$$
\begin{aligned}
& \frac{\langle c\rangle_{3}}{c_{0}}=1-8 X_{\infty} \theta \tau+O\left(\tau^{4 / 3}\right) \\
& \frac{\delta_{3}}{R}=1.536117 \tau^{1 / 3}+O\left(\tau^{2 / 3}\right) .
\end{aligned}
$$

It is interesting that leading terms (88)-(90) coincide with the corresponding expressions (73), (75) and (76) for boundary value problem of the second kind.

Figure 2 shows the dependence of $c_{w 3} / c_{0},\langle c\rangle_{3} / c_{0}$ and $\delta_{3} / R$ on $\theta$ for different values of $\tau$, and in Fig. 3 these functions are plotted vs $\tau$. Both figures indicate that the behavior of the considered functions is determined by the leading terms (88)-(90).

\section{CONCLUDING REMARKS}

Practical formulae have been derived for the mass-and heat-transfer characteristics in the entrance region of circular tubes. In particular, solutions for the problems with the boundary conditions of the first and second kind have been extended and the solution for the boundary condition of the third kind has been obtained for the first time. The case with variable diffusion coefficient, which is of practical interest, especially for multiphase flows, has been considered and its influence on the transport characteristics has been studied. The general solution can also be used, for example, to describe transport in a chemical tubular reactor; the corresponding eigenvalue solution and the first term of the asymptotic solution were obtained in [18].

The solution derived in the present paper is only valid in the mass entry region where the boundary-layer thickness is small in comparison with the tube radius. Downstream of this region and before the region with fully-developed concentration, a boundary-layer approximation is not applicable. In these regions the solution can be easily obtained by the eigenvalue method. The boundary-layer solution does not apply in the immediate vicinity of the inlet edge of the tube since the axial diffusion is not negligible in this region. The limits of validity of different approximations to the mass entry problem have been carefully analyzed in a recent paper [19].

\section{Acknowledgments}

The authors gratefully acknowledge the help of Mr. William Hawkins who did numerical computations and Mrs. Rhoda G. Miller who typed the manuscript. This work was supported by NIH grants HL 17421 and NO 1CB-63981.

\section{NOMENCLATURE}

$$
\begin{array}{ll}
A_{n}^{r}, B_{n}^{r} & \text { coefficients introduced in (29) } \\
c & \text { concentration }
\end{array}
$$




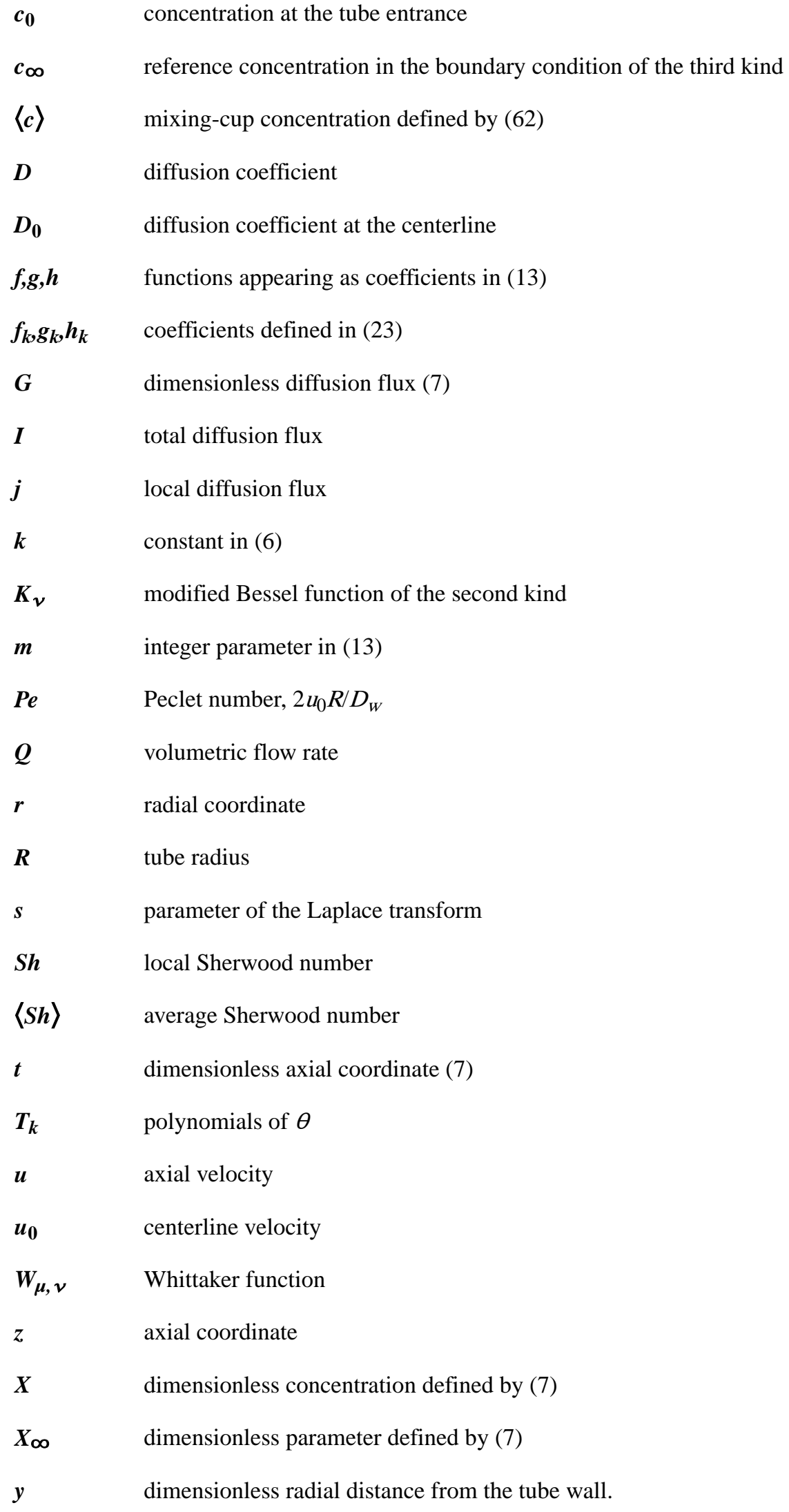




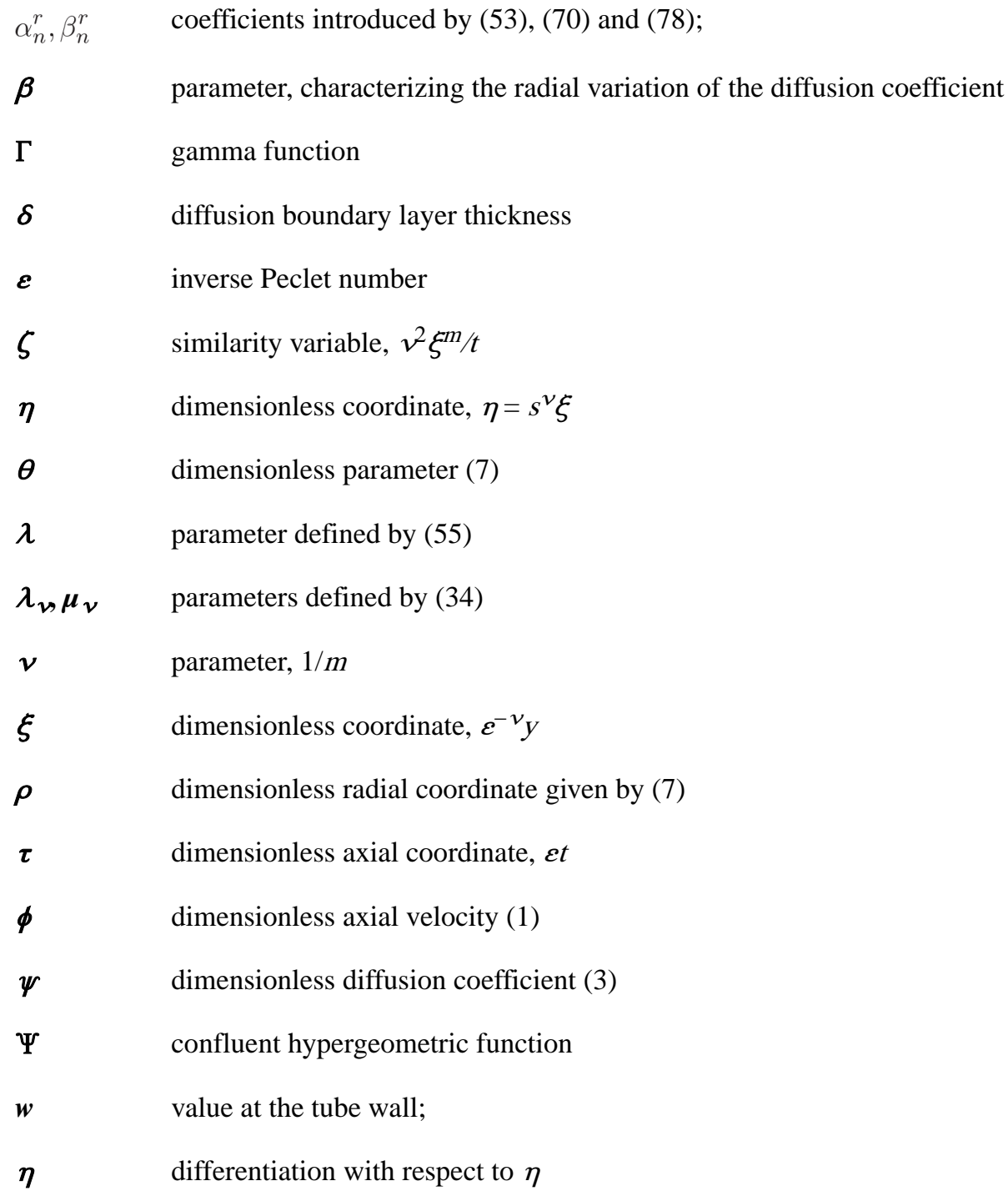

\section{References}

1. Brown GM. Heat and mass transfer in a fluid in laminar flow in a circular or flat conduit. AICh E Jl. 1960; 6:179-183.

2. Sellars JR, Tribus M, Klein JS. Heat transfer to laminar flow in a round tube or flat conduit- the Graetz problem extended. Trans Am Soc Mech Engrs. 1956; 78:441-448.

3. Davis HR, Parkinson GV. Mass transfer from small capillaries with wall resistance in the laminar flow regime. Appl Scient Res. 1970; 22:20-30.

4. Siegel R, Sparrow EM, Hallman TM. Steady laminar heat transfer in a circular tube with prescribed wall heat flux. Appl Scient Res. 1958; A7:386-392.

5. Lundberg RE, McCuen PA, Reynolds WC. Heat transfer in annular passages. Hydrodynamically developed laminar flow with arbitrarily prescribed wall temperatures or heat fluxes. Int J Heat Mass Transfer. 1963; 6:495-529.

6. Sideman S, Luss D, Peck RE. Heat transfer in laminar flow in circular and flat conduits with (constant) surface resistance. Appl Scient Res. 1964/1965; A14:157-171.

7. Mc Mercer AD. The growth of the thermal boundary layer at the inlet to a circular tube. Appl Scient Res. 1960; A9:450-456. 
8. McMercer AD. The growth of the thermal boundary layer between parallel flat plates. Appl Scient Res. 1959; A-8:357-365.

9. Lévêque MA. Les dois de la transmission de chaleur par convection. Annls Mines, Memoires, Ser 12. 1928; 13:201-415.

10. Worsøe-Schmidt PM. Heat transfer in the thermal entrance region of circular tubes and annular passages with fully developed laminar flow. Int J Heat Mass Transfer. 1967; 10:541-551.

11. Newman J. Extention of the Lévêque solution. J Heat Transfer. 1969; 91C:177-178.

12. Kooijman JM. Laminar heat or mass transfer in rectangular channels and in cylindrical tubes for fully developed flow: comparison of solutions obtained for various boundary conditions. Chem Engng Sci. 1973; 28:1149-1160.

13. Keller KH. Effect of fluid shear on mass transport in flowing blood. Fedn Proc Fedn Am Socs Exp Biol. 1971; 30:1591-1599.

14. Leal LG. On the effective conductivity of a dilute suspension of spherical drops in the limit of low Peclet number. Chem Engng Commun. 1973; 1:21-31.

15. Gradshtejn, IS., Ryzhik, IM. Tables of Integrals, Series and Products. Academic Press; New York: 1965.

16. Bateman, H., Erdélyi, A. Tables of Integral Transforms. Vol. 1. McGraw-Hill; New York: 1954.

17. Levich, VG. Physicochemical Hydrodynamics. Prentice Hall; Englewood Cliffs, N. J: 1962.

18. Lauwerier HA. A diffusion problem with chemical reaction. Appl Scient Res. 1959; A8:366-376.

19. Yao LS, Tier CL, Berger SA. Thermal analysis of a fast-moving slab in two-adjacent temperature chambers. J Heat Transfer. 1976; 98C:326-329. 


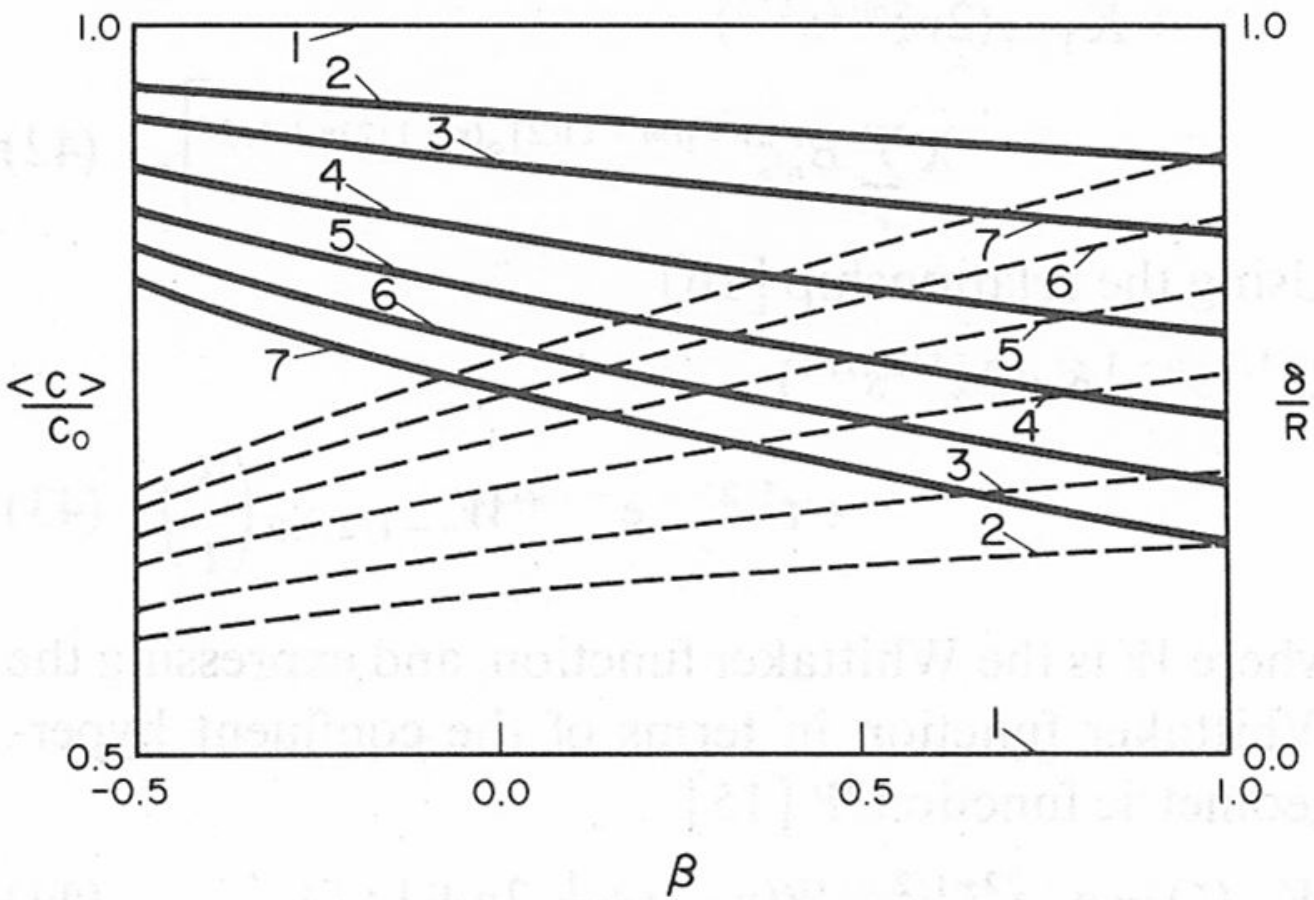

Fig. 1.

Dimensionless mixing-cup concentration $\langle c\rangle / c_{0}$ and diffusion boundary layer thickness $\delta / R$ as functions of the diffusion coefficient variability parameter $\beta$, for the boundary condition of the first kind at the tube wall; $-\langle c\rangle / c_{0},----\delta / R .1 . \tau=0,2 . \tau=10^{-3}, 3 . \tau=2 \times 10^{-3}, 4$. $\tau=4 \times 10^{-3}, 5 . \tau=6 \times 10^{-3}, 7 . \tau=10^{-2}$. 

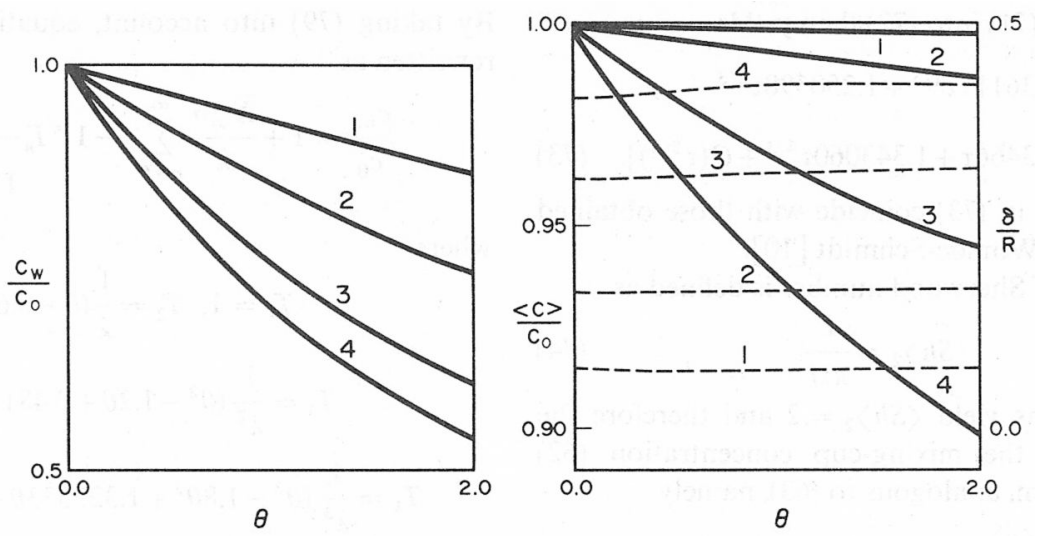

Fig. 2.

(a) Dimensionless concentration at the tube wall $c_{W} / c_{0}$, and (b) mixing-cup concentration $\langle c\rangle / c_{0}$, and diffusion boundary layer thickness $\delta / R$ vs parameter $\theta$ for the boundary condition of the third kind; $-\langle c\rangle / c_{0},----\delta / R . c_{\infty}=0,1 . \tau=10^{-4}, 2 . \tau=10^{-3}, 3 . \tau=5 \times$ $10^{-3}, 4 . \tau=10^{-2}$. 

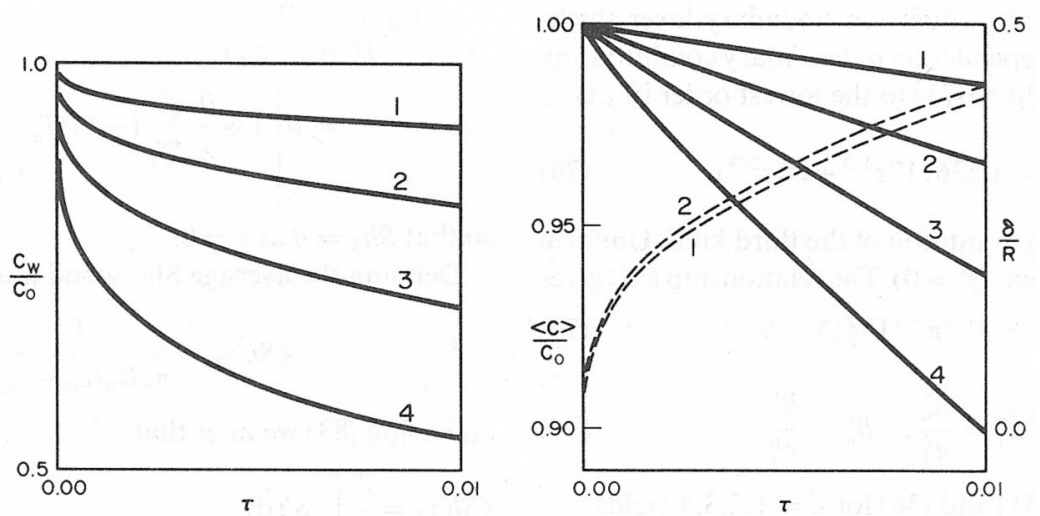

Fig. 3.

(a) Dimensionless concentration at the tube wall $c_{W} / c_{0}$, and (b) mixing-cup concentration $\langle c\rangle / c_{0}$, and diffusion boundary-layer thickness $\delta / R$ vs axial coordinate for the boundary condition of the third kind; $-\langle c\rangle / c_{0},----\delta / R . c_{\infty}=0,1 . \theta=0.2,2 . \theta=0.5,3 . \theta=1.0$, 4. $\theta=2.0$. 